\title{
Stability analysis of a three species syn-eco dynamical system with a limited alternative food for all the three species.
}

\author{
K. Kondala Rao ${ }^{1}$ \& K. Lakshmi Narayan ${ }^{2}$ \\ ${ }^{1}$ Gururnanak Institute of Technology, Ibrahimpatnam, Hyderabad-501506, \\ kkrao.kanaparthi@gmail.com \\ ${ }^{2}$ SLC'S Institute of Engineering \& Technology, Piglipur, Hyderabad-501512. \\ narayankunderu@yahoo.com
}

Keywords: Mutualism, Equilibrium points, Amensol.

\begin{abstract}
The present paper is devoted to an analytic investigation of a three species syn-eco system comprising two mutually helping species, both amensol on a third species. All possible equilibrium points are identified and their stability criteria is discussed by using Routh- Hurwitz criteria. Further, the analytical results are supported by numerical simulation using Mat Lab.
\end{abstract}

\section{Introduction.}

Every since research in the discipline of theoretical ecology was initiated by Lotka [8] and by Volterra [15], several mathematicians and ecologists contributed in the growth of this area of knowledge, which has been extensively reported in the treatises of Meyer [9], Cushing [2], Paul Conlinvaux [10], Freedman [3], Kanpur [5,6]. The ecological interactions can be broadly classified as prey-predation, competitions, neutralism, and mutualism and so on. N. C Srinivas [14] studied the competitive eco-system of two species and three species with regard to limited and unlimited resources. Later, Lakshmi Narayan [7] has investigated the two species prey-predator models. Recently stability analysis of competitive species was investigated by Archana Reddy [1]. Local stability analysis for two-species ecological mutualism model has been presented by B. Ravindra Reddy et al [11]. Recently, stability analysis of prey, two predators which are neutral to each other [12], prey, predator and super-predator [13] were carried out by Shiva Reddy and N. Ch. Pattabhi Ramacharyulu.

The present investigation is an analytical study of three species system comprising two mutualistic spices, which are amonsolon third species. The model is represented by a system of three ordinary differential equations. All possible equilibrium points are identified and their stability was discussed using Routh-Hurwitz criteria. Further solutions of quasi-linearized equations are identified and the results are simulated by Numerical examples using Mat Lab.

\section{Basic Equations.}

The basic model equations for a system of three interacting spices is given by the following set of non-linear first order simultaneous differential equations

$$
\begin{aligned}
& \text { (i) } \frac{d N_{1}}{d t}=f_{1}\left(N_{1}, N_{2}, N_{3}\right)=a_{1} N_{1}-\alpha_{11} N_{1}^{2}+\alpha_{12} N_{1} N_{2} \\
& \text { (ii) } \frac{d N_{2}}{d t}=f_{2}\left(N_{1}, N_{2}, N_{3}\right)=a_{2} N_{2}-\alpha_{22} N_{2}^{2}+\alpha_{21} N_{1} N_{2} \\
& \text { (iii) } \frac{d N_{3}}{d t}=f_{3}\left(N_{1}, N_{2}, N_{3}\right)=a_{3} N_{3}-\alpha_{33} N_{3}^{2}-\alpha_{31} N_{1} N_{3}-\alpha_{32} N_{2} N_{3}
\end{aligned}
$$

With the following notation

$N_{1}$ (t) : Population of the first species at time.

$N_{2}$ (t) : Population of the second Species at time. 
$N_{3}(\mathrm{t}):$ Population of the third Species at time.

$a_{i} \quad:$ The natural growth rates, $\mathrm{i}=1,2,3$.

$\alpha_{i i} \quad:$ The rate of decrease of Species due to its own insufficient resources $i=1,2,3$.

$\alpha_{12} \quad:$ The rate of increase of the species.

$\alpha_{21} \quad:$ The rate of increase of the species.

$\alpha_{3 i} \quad:$ The rate of decrease of the species for $\mathrm{i}=1,2$.

Further the variables $N_{1}, N_{2}, N_{3}$ are non-negative and the model parameters $a_{i}, \alpha_{i i}, i=1,2,3, \alpha_{12}, \alpha_{21}, \alpha_{3 i}, i=1,2$ are assumed to be non negative constants.

\section{Equilibrium Points.}

The system under investigation has eight equilibrium states given by $\frac{d N_{i}}{d t}=0, i=1,2,3$.

1.e., $f_{1}\left(N_{1}, N_{2}, N_{3}\right)=0 f_{2}\left(N_{1}, N_{2}, N_{3}\right)=0$ and $f_{3}\left(N_{1}, N_{2}, N_{3}\right)=0$

i. A fully washed state:

$$
\bar{N}_{1}=0 ; \bar{N}_{2}=0 ; \bar{N}_{3}=0
$$

ii. $\quad$ First and second species washed out state:

$$
\bar{N}_{1}=0, \bar{N}_{2}=0, \bar{N}_{3}=\frac{a_{3}}{\alpha_{33}}
$$

iii. First and third species washed out state:

$$
\bar{N}_{1}=0, \bar{N}_{2}=\frac{a_{2}}{\alpha_{22}}, \bar{N}_{3}=0
$$

iv. Second and third species washed out state:

$$
\bar{N}_{1}=\frac{a_{1}}{\alpha_{11}}, \bar{N}_{2}=0, \bar{N}_{3}=0
$$

v. Only first specie washed out state:

$$
\bar{N}_{1}=0, \bar{N}_{2}=\frac{a_{2}}{\alpha_{22}}, \bar{N}_{3}=\frac{\alpha_{22} a_{3}-\alpha_{32} a_{2}}{\alpha_{22} \alpha_{32}}
$$

This state would exist only when $\alpha_{22} a_{3}-\alpha_{32} a_{2}>0$

vi. Only second specie washed out state:

$$
\bar{N}_{1}=\frac{a_{1}}{\alpha_{11}}, \bar{N}_{2}=0, \bar{N}_{3}=\frac{a_{3} \alpha_{11}-a_{1} \alpha_{31}}{\alpha_{11} \alpha_{33}}
$$

This state would exist only when $a_{3} \alpha_{11}-a_{1} \alpha>0$

vii. Only third specie washed out state:

$$
\bar{N}_{1}=\frac{a_{1} \alpha_{22}+a_{2} \alpha_{12}}{\alpha_{11} \alpha_{22}-\alpha_{12} \alpha_{21}}, \bar{N}_{2}=\frac{a_{2} \alpha_{11}+a_{1} \alpha_{21}}{\alpha_{11} \alpha_{22}-\alpha_{12} \alpha_{21}}, \bar{N}_{3}=0
$$

This state would exist only when $\alpha_{11} \alpha_{22}-\alpha_{12} \alpha_{21}>0$

viii. Co-existent state or Normal steady state:

$$
\begin{aligned}
& \bar{N}_{1}=\frac{\alpha_{33}\left(a_{1} \alpha_{22}+a_{2} \alpha_{12}\right)}{\alpha_{11} \alpha_{22} \alpha_{33}-\alpha_{12} \alpha_{21} \alpha_{33}}, \bar{N}_{2}=\frac{-\alpha_{33}\left(a_{2} \alpha_{11}+a_{1} \alpha_{21}\right)}{\alpha_{11} \alpha_{22} \alpha_{33}-\alpha_{12} \alpha_{21} \alpha_{33}}, \\
& \bar{N}_{3}=\frac{-a_{1}\left(\alpha_{21} \alpha_{32}+\alpha_{22} \alpha_{31}\right)-\alpha_{11}\left(a_{2} \alpha_{32}-a_{3} \alpha_{22}\right)-\alpha_{12}\left(a_{2} \alpha_{31}+a_{3} \alpha_{21}\right)}{\alpha_{11} \alpha_{22} \alpha_{33}-\alpha_{12} \alpha_{21} \alpha_{33}}
\end{aligned}
$$

This state would be exist only when $\alpha_{11} \alpha_{22} \alpha_{33}-\alpha_{12} \alpha_{21} \alpha_{33}>0$, 


\section{Stability of the system at Equilibrium points.}

To examine the stability of the equilibrium state $\left(\bar{N}_{1}, \bar{N}_{2}, \bar{N}_{3}\right)$ we consider a small perturbation $u_{1}, u_{2}, u_{3}$, such that

$$
N_{1}=\bar{N}_{1}+u_{1}, N_{2}=\bar{N}_{2}+u_{2}, N_{3}=\bar{N}_{3}+u_{3}
$$

After linearization we get

$$
\frac{d U}{d t}=A U
$$

Where

$$
\mathrm{A}=\left[\begin{array}{ccc}
a_{1}-2 \alpha_{11} N_{1}+\alpha_{12} N_{2} & \alpha_{12} N_{1} & 0 \\
\alpha_{21} N_{2} & a_{2}-2 \alpha_{22} N_{2}+\alpha_{21} N_{1} & 0 \\
-\alpha_{31} N_{3} & -\alpha_{32} N_{3} & a_{3}-2 \alpha_{33} N_{3}-\alpha_{31} N_{1}-\alpha_{32} N_{2}
\end{array}\right]
$$

The characteristic equation for the system is $\operatorname{det}[\mathrm{A}-\lambda \mathrm{I}]=0$.

The equilibrium state is stable when the roots of the equation (4.3) are negative if they are real or have negative real parts if they are complex.

\subsection{Stability of fully washed out state:}

The stability of this equilibrium state is unstable. Since the Eigen values of the Characteristic equation are $\lambda_{1}=a_{1}, \lambda_{2}=a_{2}, \lambda_{3}=a_{3}$ all are positive.

The solution of the perturbed equations are $u_{1}=u_{10} e^{a_{1} t}, u_{2}=u_{20} e^{a_{2} t}, u_{3}=u_{30} e^{a_{3} t}$.

\subsection{Stability of first and second species washed out state:}

The stability of this equilibrium state is unstable. Since the Eigen values of the Characteristic equation are $\lambda_{1}=a_{1}, \lambda_{2}=a_{2}, \lambda_{3}=a_{3}$ all are positive.

The solution of the perturbed equations are

$$
\begin{aligned}
& u_{1}=u_{10} e^{a_{1} t}, u_{2}=u_{20} e^{a_{2} t} \\
& u_{3}=\frac{-\left(\alpha_{31} \bar{N}_{3} u_{10}\right) e^{a_{1} t}}{a_{1}+a_{3}}-\frac{\left(\alpha_{32} \bar{N}_{3} u_{20}\right) e^{a_{2} t}}{a_{2}+a_{3}}+\left(u_{30}+\frac{\alpha_{31} \bar{N}_{3} u_{10}}{a_{1}+a_{3}}+\frac{\alpha_{32} \bar{N}_{3} u_{20}}{a_{2}+a_{3}}\right) e^{-a_{3} t}
\end{aligned}
$$

\subsection{Stability of first and third species washed out state:}

The Eigen values of the characteristic equation for this state are $\lambda_{1}=a_{1}+\frac{\alpha_{12}}{\alpha_{22}} a_{2}, \lambda_{2}=-a_{2}$, $\lambda_{3}=a_{3}-\frac{\alpha_{32}}{\alpha_{22}} a_{2}$, in these clearly $\lambda_{1}$ is positive, hence the equilibrium state is unstable.

The solution of the perturbed equations are

$$
\begin{aligned}
& u_{1}=u_{10} e^{\left(a_{1}+\alpha_{12} \bar{N}_{2}\right) t}, u_{2}=\frac{\alpha_{21} \bar{N}_{2} u_{10}}{a_{1}+\alpha_{12} \bar{N}_{2}+a_{2}} e^{\left(a_{1}+\alpha_{12} \bar{N}_{2}\right) t}+\left(u_{20}-\frac{\alpha_{21} \bar{N}_{2} u_{10}}{a_{1}+\alpha_{12} \bar{N}_{2}+a_{2}}\right) e^{-a_{2} t}, \\
& u_{3}=u_{30} e^{\left(a_{3}-\alpha_{32} \bar{N}_{2}\right) t}
\end{aligned}
$$

\subsection{Stability of second and third species washed out state:}

The Eigen values of the characteristic equation for this state are $\lambda_{1}=-a_{1}, \lambda_{2}=a_{2}+\frac{\alpha_{21}}{\alpha_{11}} a_{1}$, $\lambda_{3}=a_{3}-\frac{\alpha_{32}}{\alpha_{22}} a_{1}$, in these clearly $\lambda_{2}$ is positive, hence the equilibrium state is unstable.

The solution of the perturbed equations are 


$$
\begin{aligned}
& u_{1}=\frac{\alpha_{12} \bar{N}_{1} u_{20}}{a_{1}+a_{2}+\alpha_{21} \bar{N}_{1}} e^{\left(a_{2}+\alpha_{21} \bar{N}_{1}\right) t}+\left(u_{10}-\frac{\alpha_{12} \bar{N}_{1} u_{20}}{a_{1}+a_{2}+\alpha_{21} \bar{N}_{1}}\right) e^{-a_{1} t}, u_{2}=u_{20} e^{\left(a_{2}+\alpha_{21} \bar{N}_{1}\right) t}, \\
& u_{3}=u_{30} e^{\left(a_{3}-\alpha_{31} \bar{N}_{1}\right) t}
\end{aligned}
$$

\subsection{Stability of only first specie washed out state:}

The Eigen values of the characteristic equation for this state are $\lambda_{1}=a_{1}+\alpha_{12} \bar{N}_{2}, \lambda_{2}=-a_{2}$, $\lambda_{3}=-\left(\alpha_{33} \bar{N}_{3}+\alpha_{32} \bar{N}_{2}\right)$, in these clearly $\lambda_{1}$ is positive, hence the equilibrium state is unstable.

The solution of the perturbed equations are

$$
\begin{aligned}
& u_{1}=u_{10} e^{\left(a_{1}+\alpha_{12} \bar{N}_{2}\right) t}, u_{2}=\frac{\alpha_{21} \bar{N}_{2} u_{10}}{a_{1}+\alpha_{12} \bar{N}_{2}+a_{2}} e^{\left(a_{1}+\alpha_{12} \bar{N}_{2}\right) t}+\left(u_{20}-\frac{\alpha_{21} \bar{N}_{2} u_{10}}{a_{1}+\alpha_{12} \bar{N}_{2}+a_{2}}\right) e^{-a_{2} t}, \\
& u_{3}=(-m-n) e^{\left(a_{1}+\alpha_{12} \bar{N}_{2}\right) t}-k e^{-a_{2} t}+\left(u_{30}+m+n+k\right) e^{-\alpha_{33} \bar{N}_{3} t}
\end{aligned}
$$

where

$$
l=\frac{\alpha_{21} \bar{N}_{2} u_{10}}{a_{1}+\alpha_{12} \bar{N}_{2}+a_{2}} m=\frac{\alpha_{31} \bar{N}_{3} u_{10}}{a_{1}+\alpha_{12} \bar{N}_{2}+\alpha_{33} \bar{N}_{3}}, n=\frac{\alpha_{32} \bar{N}_{3} l}{\left(a_{1}+\alpha_{12} \bar{N}_{2}+\alpha_{33} \bar{N}_{3}\right)} \& k=\frac{\alpha_{32} \bar{N}_{3}}{-a_{2}+\alpha_{33} \bar{N}_{3}}\left(u_{20}-l\right)
$$

\subsection{Stability of only second specie washed out state:}

The Eigen values of the characteristic equation for this state are $\lambda_{1}=-a_{1}, \lambda_{2}=a_{2}+\alpha_{21} \bar{N}_{1}$, $\lambda_{3}=-\alpha_{33} \bar{N}_{3}$, in these clearly $\lambda_{2}$ is positive, hence the equilibrium state is unstable.

The solution of the perturbed equations is

$$
\begin{aligned}
& u_{1}=\frac{\alpha_{12} \bar{N}_{1} u_{20}}{a_{1}+a_{2}+\alpha_{21} \bar{N}_{1}} e^{\left(a_{2}+\alpha_{21} \bar{N}_{1}\right) t}+\left(u_{10}-\frac{\alpha_{12} \bar{N}_{1} u_{20}}{a_{1}+a_{2}+\alpha_{21} \bar{N}_{1}}\right) e^{-a_{1} t}, u_{2}=u_{20} e^{\left(a_{2}+\alpha_{21} \bar{N}_{1}\right) t}, \\
& u_{3}=\left(-m_{1}-k_{1}\right) e^{\left(a_{2}+\alpha_{21} \bar{N}_{1}\right) t}-n_{1} e^{-a_{1} t}+\left(u_{30}+m_{1}+n_{1}+k_{1}\right) e^{-\alpha_{33} \bar{N}_{3} t}
\end{aligned}
$$

Where

$$
l_{1}=\frac{\alpha_{12} \bar{N}_{1} u_{20}}{a_{1}+a_{2}+\alpha_{21} \bar{N}_{1}}, m_{1}=\frac{\alpha_{31} \bar{N}_{3} l}{a_{2}+\alpha_{21} \bar{N}_{1}+\alpha_{33} \bar{N}_{3}}, n_{1}=\frac{\alpha_{31} \bar{N}_{3}\left(u_{10}-l\right)}{\left(-a_{1}+\alpha_{33} \bar{N}_{3}\right)} \& k_{1}=\frac{\alpha_{32} \bar{N}_{3} u_{20}}{a_{2}+\alpha_{21} \bar{N}_{1}+\alpha_{33} \bar{N}_{3}}
$$

\subsection{Stability of only third specie washed out state:}

One of the Eigen values of the matrix $\mathrm{A}$ is $a_{3}-\alpha_{31} \bar{N}_{1}-\alpha_{32} \bar{N}_{2}$ and the other two Eigen values are obtained from $\lambda^{2}+\left(\alpha_{11} \bar{N}_{1}+\alpha_{22} \bar{N}_{2}\right) \lambda+\left(\alpha_{11} \alpha_{22}-\alpha_{12} \alpha_{21}\right) \bar{N}_{1} \bar{N}_{2}=0$,

Whose sum $-\left(\alpha_{11} \bar{N}_{1}+\alpha_{22} \bar{N}_{2}\right)$, is always negative and their product $\left(\alpha_{11} \alpha_{22}-\alpha_{12} \alpha_{21}\right) \bar{N}_{1} \bar{N}_{2}$, is always positive. Therefore the roots of (4.7.1) are real and negative or complex conjugates having negative real parts. Thus the state is asymptotically stable only if $a_{3}<\left(\alpha_{31} \bar{N}_{1}+\alpha_{32} \bar{N}_{2}\right)$

The solution of the perturbed equations are

$$
u_{1}=A e^{-S_{1} t}+B e^{-S_{2} t}, u_{2}=A_{1} e^{-S_{1} t}+B_{1} e^{-S_{2} t}, u_{3}=u_{30} e^{\left(a_{3}-\alpha_{31} \bar{N}_{1}-\alpha_{32} \bar{N}_{2}\right) t} .
$$

Where

$$
\begin{aligned}
& A=\frac{\alpha_{21} u_{10} \bar{N}_{2}+u_{20}\left(\alpha_{11} \bar{N}_{1}-S_{1}\right)}{S_{2}-S_{1}}, B=\frac{\alpha_{21} u_{10} \bar{N}_{2}+u_{20}\left(\alpha_{11} \bar{N}_{1}-S_{2}\right)}{S_{2}-S_{1}} \\
& A_{1}=\frac{\alpha_{12} u_{20} \bar{N}_{1}+u_{10}\left(\alpha_{22} \bar{N}_{2}-S_{1}\right)}{S_{2}-S_{1}}, B_{1}=\frac{\alpha_{12} u_{20} \bar{N}_{1}+u_{10}\left(\alpha_{22} \bar{N}_{2}-S_{2}\right)}{S_{2}-S_{1}}
\end{aligned}
$$

\subsection{Stability of Co-Existing state:}

The characteristic equation of Co-existing state is

$$
\lambda^{3}+P_{1} \lambda^{2}+P_{2} \lambda+P_{3}=0
$$

Where $P_{1}=\left(\alpha_{11} \bar{N}_{1}+\alpha_{22} \bar{N}_{2}+\alpha_{33} \bar{N}_{3}\right)$ 


$$
\begin{aligned}
& P_{2}=\left(\alpha_{11} \bar{N}_{1}+\alpha_{22} \bar{N}_{2}\right) \alpha_{33} \bar{N}_{3}+\left(\alpha_{11} \alpha_{22}-\alpha_{12} \alpha_{21}\right) \bar{N}_{1} \bar{N}_{2} \\
& P_{3}=\left(\alpha_{11} \alpha_{22} \alpha_{33}-\alpha_{12} \alpha_{21} \alpha_{33}\right) \bar{N}_{1} \bar{N}_{2} \bar{N}_{3}
\end{aligned}
$$

According to Routh-Hurwitz's criteria, the necessary and sufficient conditions for stability of coexistent points are $\mathrm{P}_{1}>0, \mathrm{P}_{3}>0$ and $\left(\mathrm{P}_{1} \mathrm{P}_{2}-\mathrm{P}_{3}\right)>0$

It is evident that $P_{1}>0$ and

$$
\begin{aligned}
& P_{1} P_{2}-P_{3}=2 \alpha_{11} \alpha_{22} \alpha_{33} \bar{N}_{1} \bar{N}_{2} \bar{N}_{3}+\alpha_{11}{ }^{2} \alpha_{33} \bar{N}_{1}{ }^{2} \bar{N}_{3}+\alpha_{22}{ }^{2} \alpha_{33} \bar{N}_{2}{ }^{2} \bar{N}_{3}+\left(\alpha_{11} \bar{N}_{1}+\alpha_{22} \bar{N}_{2}\right) \alpha_{33}{ }^{2} \bar{N}_{3}{ }^{2} \\
& +\left(\alpha_{11} \alpha_{22}-\alpha_{12} \alpha_{21}\right) \alpha_{11} \bar{N}_{1}{ }^{2} \bar{N}_{2}+\left(\alpha_{11} \alpha_{22}-\alpha_{12} \alpha_{21}\right) \alpha_{22} \bar{N}_{1} \bar{N}_{2}{ }^{2}>0
\end{aligned}
$$

Hence the co-existent state is stable.

The solution of perturbation equations is:

$u_{1}=A_{2} e^{-S_{1} t}+B_{2} e^{-S_{2} t}+C_{2} e^{-S_{3} t}, u_{2}=A_{3} e^{-S_{1} t}+B_{3} e^{-S_{2} t}+C_{3} e^{-S_{3} t}, u_{3}=A_{4} e^{-S_{1} t}+B_{4} e^{-S_{2} t}+C_{4} e^{-S_{3} t}$.

Where

$$
\begin{aligned}
& A_{2}=\frac{u_{10} s_{1}^{2}\left(s_{2}-s_{3}\right)-p_{1} s_{1}\left(s_{2}-s_{3}\right)+q_{1}\left(s_{2}-s_{3}\right)}{s_{1}{ }^{2}\left(s_{2}-s_{3}\right)+s_{2}{ }^{2}\left(s_{3}+s_{1}\right)+s_{3}{ }^{2}\left(s_{1}-s_{2}\right)}, B_{2}=\frac{u_{10} s_{2}{ }^{2}\left(s_{3}-s_{1}\right)-p_{1} s_{2}\left(s_{3}-s_{1}\right)+q_{1}\left(s_{3}-s_{1}\right)}{s_{1}{ }^{2}\left(s_{2}-s_{3}\right)+s_{2}{ }^{2}\left(s_{3}+s_{1}\right)+s_{3}{ }^{2}\left(s_{1}-s_{2}\right)} \text {, } \\
& C_{2}=\frac{u_{10} s_{3}{ }^{2}\left(s_{1}-s_{2}\right)-p_{1} s_{3}\left(s_{1}-s_{2}\right)+q_{1}\left(s_{1}-s_{2}\right)}{s_{1}^{2}\left(s_{2}-s_{3}\right)+s_{2}^{2}\left(s_{3}+s_{1}\right)+s_{3}^{2}\left(s_{1}-s_{2}\right)}, A_{3}=\frac{u_{20} s_{1}^{2}\left(s_{2}-s_{3}\right)-p_{2} s_{1}\left(s_{2}-s_{3}\right)+q_{2}\left(s_{2}-s_{3}\right)}{s_{1}^{2}\left(s_{2}-s_{3}\right)+s_{2}^{2}\left(s_{3}+s_{1}\right)+s_{3}^{2}\left(s_{1}-s_{2}\right)} \\
& B_{3}=\frac{u_{20} s_{2}{ }^{2}\left(s_{3}-s_{1}\right)-p_{2} s_{2}\left(s_{3}-s_{1}\right)+q_{2}\left(s_{3}-s_{1}\right)}{s_{1}{ }^{2}\left(s_{2}-s_{3}\right)+s_{2}{ }^{2}\left(s_{3}+s_{1}\right)+s_{3}{ }^{2}\left(s_{1}-s_{2}\right)}, C_{3}=\frac{u_{20} s_{3}{ }^{2}\left(s_{1}-s_{2}\right)-p_{2} s_{3}\left(s_{1}-s_{2}\right)+q_{2}\left(s_{1}-s_{2}\right)}{s_{1}{ }^{2}\left(s_{2}-s_{3}\right)+s_{2}{ }^{2}\left(s_{3}+s_{1}\right)+s_{3}{ }^{2}\left(s_{1}-s_{2}\right)} \\
& A_{4}=\frac{u_{30} s_{1}{ }^{2}\left(s_{2}-s_{3}\right)-p_{3} s_{1}\left(s_{2}-s_{3}\right)+q_{3}\left(s_{2}-s_{3}\right)}{s_{1}{ }^{2}\left(s_{2}-s_{3}\right)+s_{2}{ }^{2}\left(s_{3}+s_{1}\right)+s_{3}{ }^{2}\left(s_{1}-s_{2}\right)}, B_{4}=\frac{u_{30} s_{2}{ }^{2}\left(s_{3}-s_{1}\right)-p_{3} s_{2}\left(s_{3}-s_{1}\right)+q_{3}\left(s_{3}-s_{1}\right)}{s_{1}{ }^{2}\left(s_{2}-s_{3}\right)+s_{2}{ }^{2}\left(s_{3}+s_{1}\right)+s_{3}{ }^{2}\left(s_{1}-s_{2}\right)} \\
& C_{4}=\frac{u_{30} s_{3}^{2}\left(s_{1}-s_{2}\right)-p_{3} s_{3}\left(s_{1}-s_{2}\right)+q_{3}\left(s_{1}-s_{2}\right)}{s_{1}{ }^{2}\left(s_{2}-s_{3}\right)+s_{2}{ }^{2}\left(s_{3}+s_{1}\right)+s_{3}{ }^{2}\left(s_{1}-s_{2}\right)}, p_{1}=\left(u_{10} \alpha_{33} \bar{N}_{3}+u_{10} \alpha_{22} \bar{N}_{2}+u_{20} \alpha_{12} \bar{N}\right), \\
& q_{1}=u_{10} \alpha_{22} \alpha_{33} \bar{N}_{2} \bar{N}_{3}+u_{20} \alpha_{12} \alpha_{33} \bar{N}_{1} \bar{N}_{3}, p_{2}=\left(u_{20} \alpha_{33} \bar{N}_{3}+u_{20} \alpha_{11} \bar{N}_{1}+u_{10} \alpha_{21} \bar{N}_{2}\right) \text {, } \\
& q_{2}=u_{20} \alpha_{11} \alpha_{33} \bar{N}_{1} \bar{N}_{3}+u_{10} \alpha_{21} \alpha_{33} \bar{N}_{2} \bar{N}_{3}, p_{3}=\left(u_{30} \alpha_{11} \bar{N}_{1}+u_{30} \alpha_{22} \bar{N}_{2}-u_{20} \alpha_{32} \bar{N}_{3}-u_{10} \alpha_{31} \bar{N}_{3}\right) \text {, } \\
& q_{3}=u_{30}\left(\alpha_{11} \alpha_{22}-\alpha_{12} \alpha_{21}\right) \bar{N}_{1} \bar{N}_{2}-u_{20}\left(\alpha_{11} \alpha_{32}+\alpha_{12} \alpha_{31}\right) \bar{N}_{1} \bar{N}_{3}-u_{10} \alpha_{22} \alpha_{31} \bar{N}_{2} \bar{N}_{3}
\end{aligned}
$$

And $\mathrm{S}_{1}, \mathrm{~S}_{2}$ and $\mathrm{S}_{3}$ are the roots (4.8.1).

\section{Global Stability.}

Theorem: The Co-existing State or Normal Steady State is Globally Asymptotically Stable.

Proof: Let us consider the Lapunov function for the interior equilibrium state is

$$
V\left(N_{1}, N_{2}, N_{3}\right)=\left\{N_{1}-\bar{N}_{1}-\bar{N}_{1} \ln \left(\frac{N_{1}}{\bar{N}_{1}}\right)\right\}+\left\{N_{2}-\bar{N}_{2}-\bar{N}_{2} \ln \left(\frac{N_{2}}{\bar{N}_{2}}\right)\right\}+\left\{N_{3}-\bar{N}_{3}-\bar{N}_{3} \ln \left(\frac{N_{3}}{\bar{N}_{3}}\right)\right\}
$$

Here

$$
\bar{N}_{1} \neq 0, \bar{N}_{2} \neq 0, \bar{N}_{3} \neq 0 \text {. }
$$

Differentiate equation (5.1) with respect to 't', we get

$$
\begin{aligned}
& \frac{d v}{d t}=\left(1-\frac{\bar{N}_{1}}{N_{1}}\right) \frac{d N_{1}}{d t}+\left(1-\frac{\bar{N}_{2}}{N_{2}}\right) \frac{d N_{2}}{d t}+\left(1-\frac{\bar{N}_{3}}{N_{3}}\right) \frac{d N_{3}}{d t} \\
& \Rightarrow \frac{d v}{d t}=\left\{\begin{array}{l}
\left(N_{1}-\bar{N}_{1}\right)\left(a_{1}-\alpha_{11} N_{1}+\alpha_{12} N_{2}\right)+\left(N_{2}-\bar{N}_{2}\right)\left(a_{2}-\alpha_{22} N_{2}+\alpha_{21} N_{1}\right) \\
+\left(N_{3}-\bar{N}_{3}\right)\left(a_{3}-\alpha_{33} N_{3}-\alpha_{31} N_{1}-\alpha_{32} N_{2}\right)
\end{array}\right\}
\end{aligned}
$$

Choose $a_{1}=\alpha_{11} \bar{N}_{1}-\alpha_{12} \bar{N}_{2}, a_{2}=\alpha_{22} \bar{N}_{2}-\alpha_{21} \bar{N}_{1}$ and $a_{3}=\alpha_{31} \bar{N}_{1}+\alpha_{32} \bar{N}_{2}+\alpha_{33} \bar{N}_{3}$ and substitute in (5.3) and simplification we get 


$$
\begin{aligned}
& \frac{d v}{d t} \leq-\left\{\begin{array}{l}
{\left[\alpha_{11}-\left(\frac{\alpha_{12}+\alpha_{21}-\alpha_{31}}{2}\right)\right]\left(N_{1}-\bar{N}_{1}\right)^{2}+\left[\alpha_{22}-\left(\frac{\alpha_{12}+\alpha_{21}-\alpha_{32}}{2}\right)\right]\left(N_{2}-\bar{N}_{2}\right)^{2}} \\
+\left[\alpha_{33}+\frac{\alpha_{31}+\alpha_{32}}{2}\right]\left(N_{3}-\bar{N}_{3}\right)^{2}
\end{array}\right\} \\
& \Rightarrow \frac{d v}{d t}<0
\end{aligned}
$$

Since all $\alpha_{11}, \alpha_{12}, \alpha_{21}, \alpha_{22}, \alpha_{31}, \alpha_{32} \& \alpha_{32}$ all are positive

Therefore the System is Globally Asymptotically Stable

\section{Numerical Examples.}

(1). Let $\mathrm{a}_{1}=3, \alpha_{11}=0.2, \alpha_{12}=0.4, \mathrm{a}_{2}=6, \alpha_{21}=0.06, \alpha_{22}=0.6, \mathrm{a}_{3}=9, \alpha_{31}=0.08, \alpha_{32}=0.05, \alpha_{33}=0.8$

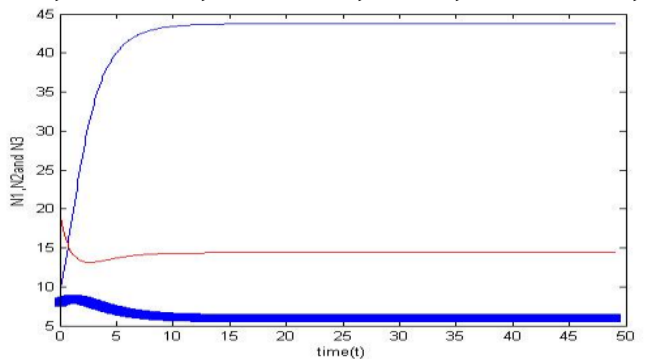

Fig 5.1.A

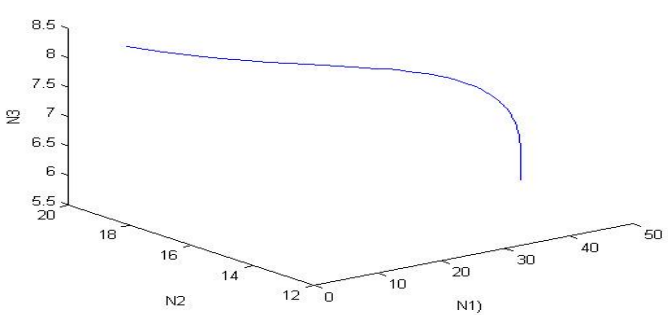

Fig 5.1.B

Figures shows the variation of the populations against the time beginning with $\mathrm{N}_{1}=9, \mathrm{~N}_{2}=20$ and $\mathrm{N}_{3}=8$.

(2). Let $\mathrm{a}_{1}=2, \alpha_{11}=0.1, \alpha_{11}=0.02, \mathrm{a}_{2}=3, \alpha_{21}=0.03, \alpha_{22}=0.1, \mathrm{a}_{3}=4, \alpha_{31}=0.01, \alpha_{32}=0.01, \alpha_{33}=0.2$.



Fig 5.2.A

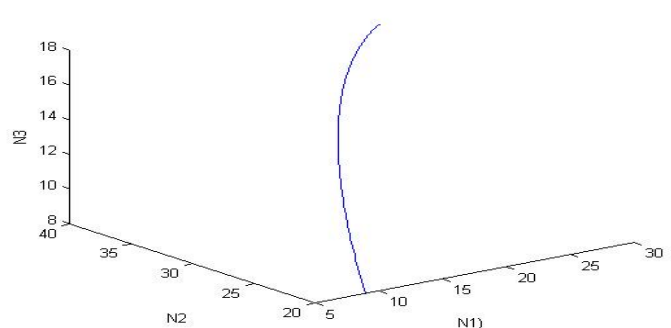

Fig 5.2.B

Figures shows the variation of the populations against the time beginning with $\mathrm{N}_{1}=9, \mathrm{~N}_{2}=20$ and $\mathrm{N}_{3}=8$.

\section{REFERENCES}

[1] Archana Reddy. R., On the stability of some mathematical models in biosciences interacting species, Ph. D thesis, JNTU 2009.

[2] Cushing, J. M., Integro-differential equations and Delay models in Population Dynamics, Lecture Notes in Biomathematics, Vol.20, Springer-Verlag, Heidelberg, 1997.

[3] Freedman. H. I., Deterministic Mathematical Models in Population Ecology, Marces Decker, New York, 1980.

[4] George F.Simmons, Differential Equations with applications and historical notes, Tata McGrawHill, New Delhi, 1974. 
[5] Kapur, J.N., Mathematical Modeling, Weley-Eastern, 1998

[6] Kapur .J.N., Mathematical Models in Biology and Medicine Affiliated East-West, 1985.

[7] Lakshmi Narayn.K., A mathematical study of Prey-Predator Ecological Models with a partial covers for the prey and alternative food for predator, Ph. D thesis, JNTU University 2004.

[8] Lokta, A.J., Elements of Physical biology, Williams and Wilkins, Baltimore, 1925.

[9] Meyer, W.J., Concepts of Mathematical Modeling, Mc Graw-Hill, 1985.

[10] Paul Colinvaux, Ecology, John Wiley and Sons Inc., New York, 1986.

[11] Ravindra Reddy.B, Lakshmi Narayn.K and Pattabhi Ramacharyulu. N. Ch., International Journal of Engg. Research and Idu.Appls, 2(II)(2009),281-291.

[12] Shiva Reddy.K, Lakshmi Narayn.K and Pattabhi Ramacharyulu. N. Ch, The International Journal of Math.Sci. \&Engg.Appls. Vol4 (Oct, 2010), pp.129-145.

[13] Shiva Reddy.K, Lakshmi Narayn.K and Pattabhi Ramacharyulu. N. Ch, The International Journal of Mathematics Applied in Science and Technology, Vol2, No.1 (2010), pp.95-107.

[14] Srinivas. N. C., Some Mathematical aspects of Modeling in Biomedical Sciences, Ph. D thesis, Kakatiya University 1991.

[15] Volterra. V, Leconssen la theory mathematique de leitte pou lavie, Gauthier-Villars, Paris, 1931. 